\title{
Crenças de auto-eficácia, autoconceito e desempenho em matemática
}

\author{
Self-efficacy beliefs, self-concept \\ and performance in mathematics
}

\author{
Liliane Ferreira Neves Inglez de SOUZA' \\ Márcia Regina Ferreira de BRITO²
}

\begin{abstract}
Resumo
Este trabalho teve como objetivo investigar as relações entre o autoconceito, as crenças de auto-eficácia e o desempenho em Matemática de alunos das séries iniciais do Ensino Fundamental. Participaram do estudo 122 estudantes de terceira e quarta séries, de ambos os gêneros, com idade variando de oito a treze anos, matriculados em uma escola pública estadual de um município do interior de São Paulo. Os dados foram coletados por meio de um questionário de auto-eficácia matemática, uma escala de autoconceito matemático e uma prova de matemática. Para se verificar correlações entre as variáveis, foi efetuada a matriz de correlação de Pearson. A análise dos resultados mostrou que o desempenho dos alunos encontrava-se positivamente relacionado ao autoconceito e à auto-eficácia matemática. Além disso, pôde-se evidenciar uma relação positiva e significativa entre tais constructos. De forma geral, os resultados deste estudo confirmaram dados de outros sobre a mesma temática, bem como as formulações teóricas da perspectiva sócio-cognitiva.
\end{abstract}

Unitermos: Auto-eficácia. Autoconceito. Desempenho. Matemática.

\begin{abstract}
The objective of the present study was to investigate the relationship between involving self-efficacy beliefs, self-concept and performance in mathematics amongst early elementary school students. The subjects comprised 122 third and fourth graders, both male and female, whose ages varied from eight to thirteen, attending a state public school in an inner city in the state of São Paulo. Data were collected using a mathematical self-efficacy questionnaire, a mathematical self-concept scale, and a mathematics test. To investigate correlations among variables, a Pearson correlation test was conducted. The results indicated that student performance was positively related to self-concept and mathematical self-efficacy. In addition, a significant, positive relationship could be seen between the beliefs of mathematical self-concept and mathematical self-efficacy. The results of the present study, in general terms, bear out other research data on the same theme, as well as theoretical assumptions from a socio-cognitive perspective.
\end{abstract}

Uniterms: Self-concept. Self-efficacy. Performance. Mathematics.

$\boldsymbol{\nabla \nabla \boldsymbol { \nabla }}$

1 Universidade Estadual de Campinas, Faculdade de Educação. Av. Bertrand Russell, 801, Cidade Universitária, "Zeferino Vaz", 13083-865, Campinas, SP, Brasil. Correspondência para/Correspondence to: L.F.N.I. SOUZA.

2 Universidade Estadual de Campinas, Faculdade de Educação, Departamento de Psicologia Educacional. Campinas, SP, Brasil.

Apoio financeiro: Fapesp - Processo no 00/03013-6. 
Na prática educacional, o desempenho acadêmico dos alunos nas diversas disciplinas escolares funciona como um indicativo de sucesso ou fracasso na aprendizagem de determinado conteúdo, podendo também apontar para a adequação ou inadequação dos diferentes métodos de ensino. Desta forma, muitos professores estão atentos ao desempenho de seus alunos, mas nem sempre têm claro conhecimento dos diversos fatores que o influenciam.

A suposição de que o desempenho resulta somente das capacidades cognitivas dos estudantes tem sido bastante questionada atualmente. Na Psicologia Educacional, além do enfoque às capacidades cognitivas e metacognitivas dos alunos, tem sido conferida uma crescente atenção ao estudo dos fatores afetivos envolvidos na aprendizagem, por acreditar-se que estes exercem um papel na motivação, no desempenho acadêmico e na futura escolha profissional dos alunos.

Assim, uma preocupação presente na educação escolar seria favorecer o desenvolvimento de auto-percepções mais favoráveis nos alunos. Quanto a este aspecto, Bandura (1986) afirmou que as práticas educacionais não deveriam ser julgadas somente pelo desenvolvimento de capacidades e conhecimentos que proporcionam, mas também pela influência que têm nas crenças das crianças sobre suas capacidades, visto que estas afetam o modo como elas enfocam o futuro.

Ao tratar do desempenho na disciplina Matemática, constructos como auto-eficácia e autoconceito têm sido considerados importantes mediadores na solução de problemas, uma vez que determinam a quantidade de tempo e esforço despendido na realização das tarefas (Pajares \& Miller, 1994).

Embora exista uma boa quantidade de pesquisas sobre esta temática em vários países, ainda são escassos os estudos no Brasil, particularmente com relação à Matemática, o que pode dificultar o acesso a este conhecimento na realidade escolar. Nesta direção, este estudo teve como objetivo verificar as relações entre o autoconceito matemático e as crenças de auto-eficácia matemática, considerando estes constructos como variáveis possivelmente relacionadas ao rendimento escolar.

\section{Autoconceito}

Bandura (1986) definiu autoconceito como"uma visão composta de um indivíduo, que é formada através

194 da experiência direta e avaliações adotadas de outras pessoas significativas" (p.409). Aparentemente, não há consenso sobre a definição deste constructo. Em uma revisão, Shavelson, Hubner e Stanton (1976) encontraram dezessete diferentes definições para o termo. Porém, segundo estes autores, em termos bem gerais, autoconceito é a percepção de uma pessoa sobre si mesma, formada e influenciada principalmente por experiências com o ambiente e outras pessoas que são significativas. Estes mesmos autores, ao listarem algumas características do autoconceito, afirmaram que este é organizado e estruturado, tem certa estabilidade, além de ser multifacetado. Pode-se considerar que a estrutura multifacetada do autoconceito segue um modelo hierárquico, que permite distinguir entre autoconceito geral, acadêmico, social, emocional e físico. O acadêmico ainda seria diferenciado dentre as várias disciplinas, podendo se considerar, por exemplo, a existência de um autoconceito matemático.

A evolução das auto-percepções está muito ligada ao desenvolvimento psicológico, que possibilita, segundo Hidalgo e Palácios (1995), a existência de um autoconceito cada vez menos global e mais diferenciado e articulado. Esses autores acrescentaram, ainda, que os avanços no desenvolvimento do conhecimento de si mesmo não ocorrem por acaso, mas estão relacionados com as crescentes capacidades cognitivas da criança e com as interações sociais que ela mantém, que são cada vez mais ricas e diferenciadas.

Shavelson et al. (1976) destacaram que as experiências de um indivíduo, em sua grande diversidade, constituem os dados nos quais ele se baseará para formar as percepções sobre si mesmo. Markus e Wurf (1987) complementam essa idéia quando afirmam que as pessoas aprendem sobre elas mesmas a partir de outros, pela comparação social e interações diretas. A respeito desta questão já afirmaram que as crianças aprendem a usar a comparação social para se avaliarem, e se tornam progressivamente mais capacitadas em fazê-lo durante os anos escolares.

Pode-se ressaltar ainda que os indivíduos atentam para aspectos pessoais que sejam distintivos em um contexto social particular (Markus \&Wurf, 1987). Por exemplo, crianças com baixo rendimento em Matemática irão atentar para seu desempenho, particularmente, se estiverem em uma classe com crianças mais habilidosas nesta disciplina. Desta forma, a comparação social pode ser uma relevante fonte de auto-conhecimento. 
Sobre esta questão, Manger e Eikelend (1997) realizaram um estudo com 924 estudantes noruegueses, relacionando desempenho e autoconceito matemático. Para fins de análise, os pesquisadores agruparam os estudantes de rendimento baixo, médio e alto em Matemática. Foram encontradas diferenças significativas entre os escores no autoconceito destes grupos. Assim, os autores apontaram que os estudantes comparam o próprio desempenho com os dos demais colegas, sendo este um indício de que a comparação social pode influenciar o autoconceito matemático. Além destes dados, um estudo longitudinal realizado por Kurt-Costez e Schneider (1994) apontou para um leve declínio do autoconceito conforme as crianças avançam em escolaridade, tornando-se as auto-percepções menos otimistas e mais realistas ao longo dos anos escolares.

Estudos sobre autoconceito acadêmico têm evidenciado relações entre este constructo e o desempenho escolar em geral (Taliuli \& Gama, 1986) e, especificamente, desempenho em Matemática (Loos, 2003), bem como entre este constructo e as crenças de autoeficácia, a motivação, o desempenho e o valor atribuído à Matemática (Shiomi, 1992). Há também evidência de que o autoconceito está negativamente relacionado à ansiedade (Bandalos, Yates \& Thorndike-Christ, 1995). $\mathrm{O}$ autoconceito ainda pode estar associado às atribuições de causalidade para sucesso e fracasso nas tarefas escolares (Chambers \& Abrami, 1991). Outro resultado, apontado pela pesquisa de Köller, Daniels, Schnabel, em 2000, realizada com 934 estudantes alemães, foi que os alunos que tinham intenção de seguir cursos relacionados à Matemática avançada apresentaram, em sua maioria, autoconceito favorável (Loos, 2003).

Evidências de pesquisas também sugerem relações entre autoconceito e dificuldades de aprendizagem, visto que estudos apontaram que crianças com dificuldades de aprendizagem apresentam menores pontuações em escalas de autoconceito (Okano, Loureiro, Linhares \& Marturano, 2004; Passeri, 2003; Pelissari, 2006; Stevanato, Loureiro, Linhares \& Marturano, 2003). Deve-se ressaltar que estes estudos não avaliaram especificamente autoconceito e desempenho em relação à Matemática, mas também podem ser úteis para se compreender a dinâmica das relações entre eles.

\section{Auto-eficácia}

O conceito de auto-eficácia foi definido como"a crença na própria capacidade de organizar e executar cursos de ações requeridas para produzir determinadas realizações" (Bandura, 1997, p.3). De forma resumida, pode-se afirmar que a auto-eficácia compreende um julgamento pessoal de capacidade relativa a um determinado domínio, e não se refere especificamente à capacidade de um indivíduo, mas sim ao que o mesmo acredita ser capaz de realizar, em uma variedade de circunstâncias (Bandura, 1986).

A auto-eficácia está vinculada às aspirações dos alunos (Bandura, Barbaranelli, Caprara \& Pastorelli, 2001) e mesmo às suas futuras escolhas profissionais. Neste sentido, Hacket e Betz (1989) realizaram um estudo com alunos universitários, verificando que estudantes que escolhem carreiras técnicas/científicas geralmente são aqueles que possuem uma forte crença em suas capacidades relativas à Matemática. Também nesta direção, O'Brien, Kopala e Martinez-Pons (1999) pesquisaram as relações entre auto-eficácia matemática e interesse por carreiras relacionadas à Matemática e às Ciências, em 415 estudantes do Ensino Médio, concluindo que a auto-eficácia matemática teve um efeito preditivo quanto ao interesse por carreiras científicas.

Em geral, a pesquisa sobre auto-eficácia tem apontado relação entre este constructo e o desempenho em Matemática (Lent, Brown \& Larkin, 1984; Pajares, 1996a; Pietsch, Walker \& Chapman, 2003; Stevens, Olivarez, Lan \& Tallent-Runnels, 2004), bem como têm sido encontradas relações entre auto-eficácia matemática e atribuições de causalidade para sucesso e fracasso nesta disciplina (Barros, 1996). Além disso, foi verificado que a auto-eficácia encontra-se negativamente relacionada com a ansiedade em relação às provas (Bandalos et al. 1995). Os resultados destas pesquisas têm ajudado a sustentar a suposição de que as crenças de auto-eficácia influenciam como as pessoas sentem, pensam, se motivam e se comportam.

A auto-eficácia produz esses efeitos diversos por meio de quatro processos principais: processos cognitivos, vinculados à antecipação de conseqüência das próprias ações, processos motivacionais, afetando a quantidade de esforço e de tempo que uma pessoa emprega em uma determinada atividade, processos afetivos, relacionados às reações emocionais dos indivíduos, stress e ansiedade que as pessoas experimentam em situações 
que consideram difíceis ou ameaçadoras, e os processos de seleção, visto que as pessoas escolhem o que vão fazer de acordo com aquilo que sentem ser capazes de executar com sucesso (Bandura, 1986, 1993, 1997; Pajares, 1996b).

Conforme observado, de acordo com o modelo sócio-cognitivo, a auto-eficácia exerce um papel na determinação do comportamento e do pensamento. Em contrapartida, alguns fatores exercem um papel na origem e no desenvolvimento destas crenças. São eles: experiências, realizações e desempenhos anteriores, experiência vicariante, persuasão social e estados fisiológicos e afetivos (Bandura, 1986, 1997).

As experiências, realizações e desempenhos anteriores constituem, segundo Bandura (1986), o mais importante fator no desenvolvimento das crenças de auto-eficácia, pois a própria experiência é a principal fonte de informação sobre as capacidades de uma pessoa. A experiência vicariante, ou seja, aquilo que se aprende a partir da experiência dos outros, também influencia como as pessoas julgam suas habilidades, pois a observação do desempenho alheio fornece informações relevantes sobre quais desempenhos um indivíduo pode realizar.

A persuasão social está fortemente vinculada às informações recebidas pelas pessoas acerca de seus desempenhos e capacidades. No contexto educacional, os alunos recebem uma grande quantidade de informações comparativas sobre suas capacidades, além de receberem notas e avaliações de professores sobre seu desempenho. Os estados fisiológicos e afetivos têm um efeito mais limitado sobre a auto-eficácia, por constituírem um fator situacional. Contudo, Bandura (1986, 1997) afirmou que as pessoas contam, em parte, com informações sobre seu estado fisiológico, e também afetivo, para julgar suas capacidades.

\section{Comparações entre os constructos}

Pode-se notar que existem semelhanças entre auto-eficácia e autoconceito, uma vez que estes dois constructos se relacionam a pensamentos auto-referentes e constituem mecanismos de agência, os quais dizem respeito, segundo Bandura (2001), a intencionalmente fazer as coisas acontecerem pelas próprias ações (p.2). Acrescenta-se que a agência relaciona-se às capacidades humanas que permitem 196 aos indivíduos exercerem algum controle sobre os eventos que afetam suas vidas, caracterizando-se por intencionalidade, capacidade preditiva, auto-reação e auto-reflexão. Considera-se, portanto, de acordo com a teoria sócio-cognitiva (Bandura, 1986) que crenças auto-referenciadas influenciam os cursos de ação que as pessoas tomam, conforme discutido anteriormente.

De acordo com Pajares (1996b), o autoconceito difere da auto-eficácia no sentido que a auto-eficácia é uma avaliação de competência para desempenhar uma determinada atividade em um contexto específico, enquanto o autoconceito pode não ser medido nesse nível de especificidade. Ele inclui crenças de auto-valorização, associadas com a competência percebida de um sujeito. Por exemplo, autoconceito matemático envolve todas as auto-percepções do aluno enquanto aprendiz desta disciplina; já a auto-eficácia diz respeito, de forma mais específica, às auto-avaliações de competência, ou ao que o aluno julga ser capaz de realizar.

Teixeira e Giacomini, em 2002, acrescentam que a auto-eficácia representa o julgamento pessoal da capacidade de planejar e executar comportamentos específicos em determinadas situações, ao passo que o autoconceito relaciona-se a uma auto-descrição mais ampla, que inclui aspectos comportamentais, cognitivos e afetivos (Silva, 2006).

Segundo Pajares e Schunk (2001), há evidência de que as crenças de auto-eficácia e o autoconceito são relacionados e influenciam o rendimento acadêmico. Esta afirmação é apontada por pesquisas (Pietsch et al. 2003; Shiomi, 1992) que, de modo geral, têm confirmado as formulações teóricas de que o desempenho acadêmico é resultado de uma diversidade de fatores, inclusive autoconceito e auto-eficácia.

\section{Método}

\section{Participantes}

Os participantes deste estudo constituem uma amostra de conveniência, sendo 122 alunos de terceira e quarta séries da primeira fase do Ensino Fundamental, com idade variando de 8 a 13 anos (Média - M=9,52 anos; desvio-padrão - $\mathrm{DP}=0,7)$. Dentre os participantes, 63 eram do gênero masculino e 59 do gênero feminino, sendo que 50 alunos eram de terceira e 72 de quarta série. Estes alunos encontravam-se regularmente matricu- 
lados em uma escola pública estadual localizada próximo à região central de um município do interior do estado de São Paulo.

\section{Instrumentos}

1) Questionário de auto-eficácia: este questionário (Neves \& Brito, 2001) contém treze itens versando sobre a confiança dos alunos relativa à realização de diversas atividades relacionadas à aprendizagem da Matemática. No início do questionário, o aluno é solicitado a indicar o quanto está confiante de que é capaz de realizar as atividades com sucesso. Cada item do instrumento diz respeito a uma atividade, como: resolver problemas de Matemática, fazer contas, fazer uma prova, entender as explicações da professora, dentre outras.

Foi utilizada uma escala do tipo Likert, com cinco pontos, que variavam de "nada confiante"a"totalmente confiante". Para as alternativas de resposta, foram utilizadas ilustrações que demonstravam, por expressões faciais, os graus de confiança, sendo que as mesmas constavam como parte das respostas. Foi realizado um estudo de validação do instrumento (Neves, 2002), com 397 estudantes de escolas públicas, das mesmas séries escolares, de ambos os gêneros, com idade variando de 8 a 15 anos. A análise estatística dos dados obtidos neste estudo de validação mostrou que o instrumento obteve um coeficiente alfa de 0,88, indicando forte consistência interna. Na presente amostra, o questionário obteve o coeficiente alfa de 0,89.

2) Escala de autoconceito matemático: a escala de autoconceito é parte de um instrumento utilizado por Pajares e Miller (1994) e contém 21 itens de escala tipo Thurstone, variando de totalmente falsa (1 ponto) a totalmente verdadeira (8 pontos). As proposições versam sobre aspectos do autoconceito matemático, por exemplo: Em comparação com os meninos da minha classe, eu sou bom em Matemática. Ou ainda: Eu tenho boas notas em Matemática. Na presente amostra, esta escala obteve um coeficiente alfa de 0,90, indicando forte consistência interna. $O$ instrumento original, no idioma inglês, foi desenvolvido e validado nos Estados Unidos, portanto este foi traduzido e adaptado. Anteriormente à aplicação nesta amostra, a escala foi aplicada em um grupo de 66 estudantes com características similares, para avaliar se estes encontravam dificuldades na leitura e compreensão dos itens do instrumento.
Solicitou-se aos mesmos que indicassem caso não entendessem algo na redação dos itens ou na forma de responder ao instrumento. Verificou-se, neste estudo preliminar (Neves, 2002), que o instrumento estaria adequado às características dos participantes.

Silva (2006) adaptou e validou este instrumento para a disciplina estatística, substituindo o termo Matemática por Estatística (exemplo: eu tenho boas notas em Estatística). Neste estudo, com 510 estudantes universitários, obteve-se um coeficiente alfa de 0,94, e a análise fatorial apontou para a unidimensionalidade do instrumento.

3) Prova de matemática: a prova de matemática foi elaborada de acordo com orientações de professores do segundo ciclo do Ensino Fundamental e de professores de Matemática. Nenhum destes teve alunos participando na pesquisa de que trata este artigo. Os problemas também foram baseados em questões apresentadas nos livros didáticos. Foram utilizadas 12 questões fechadas de múltipla escolha, com somente uma possibilidade de resposta, isto é, uma única alternativa correta. Foram elaborados dois tipos de provas: uma para terceira e outra para quarta série. Este instrumento foi testado em um estudo preliminar (Neves, 2002) a partir do qual foram feitas pequenas modificações na redação de alguns problemas, embora não tenha sido alterada a estrutura dos mesmos. Deve-se ressaltar que somente buscou-se, com isso, refinar a redação do instrumento.

\section{Procedimentos}

Todos os instrumentos foram aplicados coletivamente, em período normal de aula. Foi realizado um estudo preliminar com 66 alunos das mesmas séries escolares, de uma escola com características similares àquela na qual se realizou a coleta de dados. Este indicou a adequação dos instrumentos aos objetivos do estudo, e a possibilidade de aplicação coletiva dos mesmos.

Para garantir uma melhor compreensão da proposta dos instrumentos, eles foram lidos e as questões cuidadosamente explicadas, bem como o modo de respondê-las. Ao responderem aos instrumentos, os alunos foram supervisionados, para evitar que copiassem as respostas dos colegas. Outro cuidado tomado foi explicitar aos alunos que as pontuações obtidas nos instrumentos, mesmo na prova de Matemática, não constariam como nota escolar. 
Deve-se ressaltar que a participação foi voluntária e que os objetivos da pesquisa foram esclarecidos aos participantes. O projeto de pesquisa foi apresentado à direção da escola, esclarecendo os objetivos e os procedimentos para coletar os dados. Além disso, foi assegurado que a pesquisa não acarretaria em nenhum prejuízo para os estudantes, e que os mesmos poderiam desistir da participação. Desta forma, obteve-se um consentimento da direção da escola para a realização da pesquisa.

\section{Resultados}

Inicialmente será apresentada uma análise descritiva dos resultados da pesquisa e, a seguir, as correlações efetuadas entre as variáveis de interesse. Esta última etapa da análise foi efetuada utilizando-se a matriz de correlação de Pearson. Para este teste estatístico, foi adotado nível de significância de 0,05.

As pontuações na escala de autoconceito poderiam variar de 21 a 168 pontos, sendo que a soma de pontos obtida pelos alunos desta amostra variou de 44 a 167, com média igual a 134,4 pontos e desvio-padrão 21,1. Dado que o ponto médio da escala é 94,5, pode-se inferir que os alunos demonstraram, em sua maioria, pontuações acima da média, indicando autoconceito favorável.

A atribuição de valores de um (totalmente falsa) a oito (totalmente verdadeira) às respostas dos participantes referentes às 21 proposições da escala de autoconceito possibilitou algumas inferências sobre este constructo, no que se refere à disciplina de Matemática. Os dados indicaram que as proposições nas quais foram apresentadas maiores médias de pontos foram "Para mim é importante ter boas notas em matemática" $(M=7,6$ e DP=1,13) e"Ser bom em matemática é importante para mim" (M=7,6 e DP=0,88). A menor média de pontos foi encontrada na questão "Acredito que posso ser um matemático ou um cientista quando crescer" (M=5,2 e DP=2,49).

Neste questionário, a pontuação poderia variar de 13 a 65 pontos, sendo que a soma de pontos obtida pelos estudantes dessa amostra variou de 26 a 65, com média igual a 53,9 pontos e desvio-padrão 7,94.

Foram atribuídos valores de um (nada confiante) a cinco (totalmente confiante) às respostas dos alunos referentes às indicações de confiança expressas nas respostas dadas às 13 questões propostas no instrumento. Os resultados indicaram que os alunos apresentaram maiores pontuações na questão referente a fazer contas de somar ( $M=4,8$ e $D P=0,50)$, e menores pontuações na questão relativa a fazer uma prova de Matemática $(M=3,7$ e $D P=1,02)$.

Os dados referentes ao desempenho em Matemática foram coletados por meio de uma prova de 12 questões de múltipla escolha. Para se computar as notas na prova de Matemática, foi atribuído um ponto para cada questão correta, de modo que, se o aluno acertasse todas as questões, obteria 12 pontos. Em seguida estas pontuações foram transformadas em notas, de zero a dez. Na presente amostra, as notas obtidas pelos participantes variaram de 0,83 a 10,00, e a média geral das notas foi 5,47 (DP=2,40). Foi possível verificar que a média dos alunos da terceira série $(M=5,57$ e $D P=2,66)$ foi ligeiramente superior à média dos alunos da quarta série ( $M=5,4$ e $D P=2,20)$, mas a análise de variância (ANOVA) não apontou diferenças estatisticamente significativas entre estas médias.

Os resultados apresentados foram obtidos por meio das pontuações na escala de autoconceito e no questionário de auto-eficácia, além das pontuações referentes ao desempenho dos alunos na prova de Matemática. A análise dos resultados, efetuada a partir da matriz de correlação de Pearson, indicou relações positivas e significativas, porém moderadas, entre auto-eficácia e desempenho $(r=0,444 ; p<0,001)$, e também entre autoconceito e desempenho $(r=0,459 ; p<0,001)$.

Foi possível, ainda, verificar a existência de uma relação significativa e forte entre autoconceito matemático e crenças de auto-eficácia matemática ( $r=0,767$ e $p<0,001$ ). Estes resultados permitem a inferência de que quanto mais favoráveis o autoconceito ea autoeficácia matemática, melhor o desempenho na prova da Matemática. Os dados referentes a estas corre-lações podem ser visualizados na Tabela 1.

\section{Discussão}

Pode-se perceber, pelas pontuações nos instrumentos, que grande parte dos participantes demonstrou autoconceito favorável, além de um nível adequado de auto-eficácia. Os dados indicaram também que, quanto 
Tabela 1. Correlações entre autoconceito, auto-eficácia e desempenho em matemática.

\begin{tabular}{llc}
\hline Variáveis correlacionadas & $\begin{array}{c}\text { Correlação de } \\
\text { Pearson (r) }\end{array}$ \\
\hline Autoconceito matemático & $\begin{array}{l}\text { Auto-eficácia } \\
\text { matemática } \\
\text { Desempenho em }\end{array}$ & $0,767^{* * *}$ \\
Auto-eficácia matemática & $\begin{array}{l}\text { matemática } \\
\text { Desempenho em } \\
\text { matemática }\end{array}$ & $0,444^{* * *}$ \\
\hline
\end{tabular}

${ }^{* * *} p>0,001$.

maiores as crenças de auto-eficácia, melhor o desempenho em matemática, similarmente aos resultados encontrados por Barros (1996), Hackett e Betz (1989), Lent et al. (1984), Pajares (1996a), Shiomi (1992), Randhawa, Beamer e Lundberg (1993). Trata-se de estudos internacionais, realizados em diversos níveis escolares, porém, também estão de acordo com o estudo brasileiro de Medeiros, Loureiro, Linhares e Marturano (2000), conduzido com crianças das séries iniciais do Ensino Fundamental.

Quanto ao autoconceito matemático, repetindo dados de outras pesquisas, (Shiomi, 1992; Bandalos et al., 1995), encontrou-se uma relação positiva entre este constructo e a auto-eficácia. Este resultado está compatível com o modelo teórico sócio-cognitivista, segundo o qual os julgamentos de um indivíduo acerca de suas próprias capacidades constituem um dos aspectos de seu autoconceito.

Considera-se a existência de uma relação recíproca entre os constructos estudados. É possível afirmar que, quanto mais favoráveis a auto-eficácia e o autoconceito, maior a probabilidade de um bom desempenho. No entanto, não se propõe uma direção de causalidade em que auto-eficácia e autoconceito resultem em meIhor performance. Ao contrário, considera-se que a interação entre estes fatores é bem complexa, visto que desempenhos anteriores também afetam as auto-percepções (Bandura 1986; 1997). Desta forma, não se deve priorizar um enfoque que considere somente as capacidades cognitivas do aluno referentes à aprendizagem da Matemática como determinantes do desempenho acadêmico nesta disciplina.

Tem-se reconhecido, como um objetivo da educação escolar, promover a autonomia dos alunos quanto aos próprios processos de aprendizagem. Neste sentido, os aspectos referentes às auto-percepções, englobando as crenças acerca das próprias capacidades, desempenham um papel essencial no desenvolvimento dessa autonomia, bem como na motivação do aluno. Desta forma, a educação escolar tem um papel inestimável no estabelecimento destas crenças, tornando-se possível intervir para a modificação das crenças de alunos pouco confiantes. É conveniente lembrar que a auto-eficácia é formada a partir de quatro fontes de informação: experiências e desempenhos anteriores, experiência vicariante, persuasões verbais e estados fisiológicos. Portanto, modificações somente são possíveis atuando-se nestes aspectos, conforme exposto a seguir.

De acordo com Bandura (1986), a principal fonte de informação de eficácia são os desempenhos já apresentados pelas pessoas. Mas um estudo conduzido por Zeldin e Pajares (2000), tendo como participantes mulheres envolvidas em carreiras tecnológicas, mostrou que não são somente estas experiências que têm um poder preditivo em relação ao desenvolvimento da auto-eficácia. Ao contrário, os autores verificaram que persuasões verbais e experiências vicariantes foram os principais fatores no desenvolvimento destas crenças entre os sujeitos estudados.

No contexto escolar, a persuasão verbal seria efetuada por meio das informações transmitidas aos alunos sobre seu desempenho e sobre suas capacidades. Assim, o tipo de feedback fornecido aos alunos sobre seus desempenhos representaria uma importante forma de intervir nas crenças dos alunos a respeito de suas capacidades. É notado que os alunos recebem uma grande quantidade de informações sobre os resultados alcançados. Na maioria das vezes, essas informações são socialmente referenciadas, ou seja, o desempenho de um aluno é comparado com o desempenho de seus colegas. No entanto, o estudo realizado por Shih e Alexander (2000) indicou que crianças que recebiam feedback auto-referenciado (comparando desempenho atual com resultados anteriores) apresentaram, após algum tempo, um aumento no nível de auto-eficácia.

É certo que, no estudo de que trata este artigo, os alunos apresentaram crenças favoráveis com relação às próprias capacidades e desempenho, mas, ainda assim, é possível agir no sentido de manter favoráveis as auto-percepções destes alunos no decorrer dos anos escolares. Esta preocupação se torna justificável diante de resultados de estudos longitudinais (Kurt-Costez \& Schneider, 1994; Wigfield et al., 1997), os quais mostraram 
que as crenças das crianças referentes às próprias capacidades acadêmicas tendem a declinar no decorrer das séries escolares. Parece haver um consenso, na literatura, de que para se compreender as causas do desempenho em Matemática, bem como nas outras disciplinas, devem ser levados em conta fatores cognitivos, afetivos e motivacionais.

Especificamente com relação à Matemática, Wolters e Pintrich (1998) argumentaram que os padrões motivacionais são menos adaptativos nesta disciplina do que em outras, além de níveis altos de ansiedade estarem freqüentemente presentes em um número considerável de estudantes. Em parte, isto pode resultar da crença de que a Matemática é uma disciplina difícil de aprender e acessível a uma minoria, sendo apontada como causa de fracasso entre muitos alunos (Pirola \& Brito, 2001). Assim, professores que sustentam esta crença dificilmente terão expectativas altas com todos os alunos, e podem ensinar Matemática acreditando que somente uma parcela dos alunos irá entender (Inglez de Souza, 2006).

\section{Considerações Finais}

De acordo com a perspectiva sócio-cognitivista, existe uma relação recíproca entre desempenho e crenças auto-referenciadas. Nesta perspectiva, a posse de habilidades, isoladamente, não explica a causa de desempenhos adequados ou inadequados no contexto escolar. Neste sentido, é importante que educadores possam atentar para aspectos como crenças pessoais de capacidade, buscando favorecer o desenvolvimento de auto-percepções positivas.

De forma geral, os resultados deste estudo, referentes às relações entre autoconceito matemático, crenças de auto-eficácia e desempenho em Matemática, confirmam dados de outros estudos, citados anteriormente, bem como as formulações teóricas da perspectiva sócio-cognitiva.

A investigação realizada atendeu aos objetivos propostos, no entanto, uma de suas limitações é que a amostra se restringe a crianças de Ensino Fundamental, pertencentes a uma única escola. Portanto, um aprofundamento do estudo é necessário para que se possibilite a generalização dos resultados a outras amostras.

Além disso, por não ter sido contemplado como

200 um objetivo do estudo, os dados não permitem uma compreensão de como estas crenças auto-referenciadas se formam. Assim, um aspecto que poderia ser considerado em futuras investigações seria o desenvolvimento do autoconceito e da auto-eficácia matemática dos estudantes, visto que este conhecimento poderia contribuir para uma compreensão mais global de como as crenças auto-referenciadas dos alunos se estabelecem no decorrer dos anos escolares.

\section{Referências}

Bandalos, D. L., Yates, K., \& Thorndike-Christ, T. (1995). Effects of mathematics self-concept, perceived self-efficacy, and attributions for failure and success on test anxiety. Journal of Educational Psychology, 87 (4), 611-623.

Bandura, A. (1986). Social foundations of thought and action: a social cognitive theory. Englewood Cliffs, N. J.: Prentice-Hall.

Bandura, A. (1993). Perceived self-efficacy in cognitive development and functioning. Educational Psychologist, 28 (2), 117-148.

Bandura, A. (1997). Self-efficacy: the exercise of control. New York: Freeman.

Bandura, A. (2001). Social cognitive theory: an agentic perspective. Annual Review of Psychology, 52, 1-26.

Bandura, A., Barbaranelli, C., Caprara, G. V., \& Pastorelli, C. (2001). Self-efficacy beliefs as shapers of children's aspirations and career trajectories. Child Development, $72(1), 187-206$

Barros, A. (1996). Atribuições causais e expectativa de controlo na realização Matemática. Psychologica, (15), 135-146.

Chambers, B., \& Abrami, P. C. (1991). The relashionship between student team learning outcomes and achievement, causal attributions and affect. Journal of Educational Psychology, 83 (1), 140-146.

Hackett, G., \& Betz, N. E. (1989). An exploration of the mathematics self-efficacy/ mathematics performance correspondence. Journal for Research in Mathematics Education, 20 (3), 261-273.

Hidalgo, V., \& Palácios, J. (1995). O Desenvolvimento da personalidade dos 6 aos 12 anos. In C. Coll, J. Palácios \& A. Marchesi (Orgs.), Desenvolvimento psicológico e educação (Vol.1, pp.243-249). Porto Alegre: Artes Médicas.

Inglez de Souza, L. F. N. (2006). Crenças de auto-eficácia matemática. In R. G. Azzi \& S. A. J. Polidoro (Orgs.), Auto-eficácia em diferentes contextos (pp.111-126). Campinas: Alínea.

Kurtz-Costes, B. E., \& Schneider, W. (1994). Self-concept, attributional beliefs, and school achievement: a longitudinal analysis. Contemporary Educational Psychology, 19 (1), 199-216.

Lent, R. W., Brown, S. D., \& Larkin, K. C. (1984). Relation of self-efficacy expectations to academic achievement and 
persistence. Journal of Counseling Psychology, 31 (3), 356-362.

Loos, H. (2003). Atitudee desempenho em matemática, crenças auto-referenciadas e familia: uma path analysis. Tese de doutorado não-publicada, Faculdade de Educação, Universidade Estadual de Campinas.

Manger, T., \& Eikeland, O. J. (1997). The effect of social comparison on mathematics self-concept. Scandinavian Journal of Psychology, 38 (3), 237-241.

Markus, H., \& Wurf, E. (1987). The dynamic self-concept: A social psychological perspective. Annual Review of Psychology, 38, 299-337.

Medeiros, P. C., Loureiro, S. R., Linhares, M. B. M., \& Marturano, E. M., (2000). A auto-eficácia e os aspectos comportamentais de crianças com dificuldade de aprendizagem. Psicologia: Reflexão e Crítica, 13 (3), 327-336.

Neves, L. F. (2002). Um estudo sobre as relações entre a percepção e as expectativas de professores e dos alunos e o desempenho em matemática. Dissertação de mestrado não-publicada, Faculdade de Educação, Universidade Estadual de Campinas.

Neves, L. F., \& Brito, M. R. F. (2001). Relações entre auto-eficácia matemática e desempenho em matemática de alunos do ensino fundamental. Resumos de Comunicações Científicas, XXXIReunião Anual de Psicologia (p.198). Rio de Janeiro.

O'Brien, V., Kopala, M. \& Martinez-Pons, M. (1999). Mathematics self-efficacy, ethnic identity, gender, and career interests related to Mathematics and Science. The Journal of Educational Research, 92 (4), 232-236.

Okano, C. B., Loureiro, S. R., Linhares, M. B. M., \& Marturano, E. M. (2004). Crianças com dificuldades escolares atendidas em programa de suporte psicopedagógico na escola: avaliação do autoconceito. Psicologia Reflexão e Crítica, 17 (1), 121-128.

Pajares, F. (1996a). Self-efficacy beliefs and mathematical problem-solving of gifted students. Contemporary Educational Psychology, 21 (3), 325-344.

Pajares, F. (1996b). Self-efficacy beliefs in academic settings. Review of Educational Research, 66 (4), 543-578.

Pajares, F., \& Miller, M. D. (1994). Role of self-efficacy and self-concept beliefs in mathematical problem solving: a path analysis. Journal of Educational Research, 86 (2), 193-203.

Pajares, F., \& Schunk, D. H. (2001). Self-Beliefs and school success:Self-Efficacy, self-concept, and school Achievement. Retrieved April 11, 2002, from http://www.emory.edu/ EDUCATION/ mfp/effpage.html

Passeri, S. M. R. R. (2003). O autoconceito e as dificuldades de aprendizagem no regime de progressão continuada. Tese de doutorado não-publicada, Faculdade de Educação, Universidade Estadual de Campinas.

Pelissari, A. R. M. S. (2006). Dificuldades de aprendizagem em escrita, autoconceito e aceitação social. Tese de doutorado não-publicada, Faculdade de Educação, Universidade Estadual de Campinas.

Pietsch, J., Walker, R., \& Chapman, E. (2003). The relation among self-concept, self-efficacy, and performance in
Mathematics during secondary school. Journal of Educational Psychology, 95 (3), 589- 603.

Pirola, N. A., \& Brito, M. R. F. A (2001). Formação dos conceitos de triângulo e paralelogramo em alunos da escola elementar. In M. R. F. Brito (Org.), Psicologia da educação matemática: teoria e pesquisa (pp.85-106). Florianópolis: Insular.

Randhawa, B. S., Beamer, J. E., \& Lundberg, I. (1993). Role of mathematics self-efficacy in the structural model of mathematics achievement. Journal of Educational Psychology, 85 (1), 41-48.

Shavelson, R. J., Hubner, J. J., \& Stanton, G. C. (1976). Self-concept: validation of construct interpretations. Review of educational Research, 46 (3), 407-441.

Shih, S., \& Alexander, J. M. (2000). Interacting effects of goal setting and self- or other-referenced feedback on childrens's development of self-efficacy and cognitive skill within the Taiwanese classroom. Journal of Educational Psychology, 92 (3), 536-543.

Shiomi, K. (1992). Association of attitude toward mathematics with self-efficacy, causal atribution, and personality traits. Perceptual and Motor Skills, 75 (3), 563-567.

Silva, M. C. R. (2006). Evidências de validade de uma escala de autoconceito acadêmico em Estatística. Dissertação de mestrado não-publicada. Faculdade de Psicologia, Universidade São Francisco, Itatiba.

Stevanato, I. S., Loureiro, S. R., Linhares, M. B. M., \& Marturano, E. M. (2003). Autoconceito de crianças com dificuldades de aprendizagem e problemas de comportamento. Psicologia em Estudo, 8 (1), 67-76.

Stevens, T. Olivarez, A., Lan W. Y., \& Tallent-Runnnels, M. K. (2004). Role of mathematics self-efficacy and motivation in Mathematics performance across ethnicity. The Journal of Educational Research, 97 (4), 208-221.

Taliuli, N., \& Gama, E. M. P. (1986). Causal attribution, self-concept and academic achievement of children from IOW SES families. Trabalho apresentado no Educational Research in the Global Community: Annual Meeting of The American Educational Research Association, San Francisco CA, disponível em http://www.eric.ed.gov

Wigfield, A., Harold, R. D., Freedman-Doan, C., Eccles, J. S., Yoon, K. S., Arbreton, A. J. A., \& Blumenfeld, P. C. (1997). Change in children's competence beliefs and subjective task values across the elementary school years: a 3-year study. Journal of Educational Psychology. 89 (3), 451-469.

Wolters, C. A., \& Pintrich, P. R. (1998). Contextual differences in student motivation and self-regulated learning in mathematics, English, and social studies classrooms. Instructional Science, 26 (1), 27-47.

Zeldin, A. Z., \& Pajares, F. (2000). Against the odds: Self-efficacy beliefs of women in mathematical, scientific, and technological careers. American Educational Research Journal, 37 (1), 215-246.

Recebido em: 27/7/2006

Versão final reapresentada em: 17/5/2007

Aprovado em: 11/9/2007 